\title{
Desynchronization of clocks in rigid acceleration and gravitational redshift
}

\author{
İnanç Şahin ${ }^{a *}$ \\ ${ }^{a}$ Department of Physics, Faculty of Sciences, Ankara University, Ankara, Turkey
}

\begin{abstract}
In this paper, we examine the prediction of the theory of relativity for the desynchronization of accelerating clocks separated by a proper distance $\ell$. We adopt the approach of Larmor-LorentzPoincaré-Bell to relativity and derive clock desynchronization as a result of an acceleration procedure based on two basic assumptions. We show that contrary to expectations, as a result of acceleration, the rear clock actually shifted backwards with respect to the front clock according to an inertial observer. However, due to Einstein's equivalence principle the accelerating observer feels a gravitational field and observes that the rear clock undergoes a gravitational redshift relative to the front clock. This gravitational time shift is larger than the time shift for the acceleration and the difference is exactly equal to the special relativistic time shift. Eventually, we arrive at the conclusion that Einstein's equivalence principle and gravitational redshift is necessary to explain special relativistic clock desynchronization.
\end{abstract}

Keywords: Relativity of simultaneity, Accelerated observers, Gravitational redshift.

\section{Introduction}

Rigid acceleration is a type of acceleration in which the proper lengths in the accelerating frame remain the same. Therefore, in such an acceleration, the rigidity of a body is maintained, it does not deform or change shape in its rest frame due to the acceleration forces. In this paper, we examine the desynchronization of rigidly accelerating clocks separated by a proper distance $\ell$. We adopt the approach of Larmor-Lorentz-Poincaré-Bell to relativity [1] and derive clock desynchronization as a result of some underlying process. As it was pointed out by Bell [1], Einstein's approach to relativity differs from that of Lorentz and others in two major ways. There is a difference of philosophy, and a difference of style. The difference of style is that Einstein started from the assumption that laws would appear the same to all inertial observers. But he did not infer observers' experiences from underlying physical processes. Einstein did not create a theory of clocks and duration from first principles. The properties of clocks were not deduced from the inner structure of the theory, but were simply required to accord with the relativity principle [2]. Einstein's approach permits a very concise and elegant formulation of the theory, however his approach leaves some details unanswered. For instance, consider spatially seperated clocks with a proper distance $\ell$ synchronized in an inertial frame $S_{0}$. Then, they are accelerated into another inertial frame say $S_{1}$ which has a relative speed $v$

\footnotetext{
*inancsahin@ankara.edu.tr
} 
with respect to $S_{0}$. From Lorentz transformations we conclude that according to an observer in $S_{0}$ the trailing clock will shift forward $\Delta t=\beta \gamma \ell / c$ relative to the front clock. However according to Lorentz transformations, this time shift is a relational quantity between two clocks. Einstein's approach does not tell us whether the trailing clock has shifted forward or whether the forward clock has shifted backward. On the other hand, the details of the acceleration procedure give an answer. We show that as a result of rigid acceleration, the trailing clock actually shifted backwards. ${ }^{1}$ But, the accelerating observer $S^{\prime}$ feels a gravitational field equivalent to the acceleration and observes that the trailing clock undergoes a gravitational redshift relative to the front clock. This gravitational "backward-time shift" is larger than the time shift for the rigid acceleration and the difference is exactly equal to the special relativistic shift $\Delta t=\beta \ell / c$. Therefore, trailing clock shifts forward relative to the front clock.

As we have discussed Einstein's approach contains some freedom regarding clock synchronization: Does the trailing clock shift forward or the clock in the front shift backward? Or do both clocks shift at certain amounts? Some authors have proposed their own synchronization hypothesis, exhausting this freedom in Einstein's approach [3, 4, 5, 6]. For example, according to Kowalski's synchronized clock hypothesis $(\mathrm{SCH})$ spatially separated clocks, which are synchronized in one inertial frame, maintain their synchronization in another inertial frame as long as their proper separation remains the same when they come to rest in any other inertial frame [3, 5]. A consequence of SCH is that there is no redshift between spatially separated clocks which are rigidly accelerating. On the other hand, if we consider the details of the acceleration procedure and derive the clock desynchronization as the result of this procedure, we realize that $\mathrm{SCH}$ will not be valid. ${ }^{2}$

We will make two basic assumptions during our calculations: (i) The interactions leading to acceleration occur simultaneously at different points in the accelerating frame, relative to an observer in that frame. We assume that the acceleration takes place by elementary particle interactions. These interactions occur simultaneously in the accelerating frame. This assumption is necessary for the acceleration to be rigid. Otherwise, the acceleration forces deform the accelerating body and change the proper lengths. For instance, assume that the accelerating body is accelerating due to electromagnetic forces. Then, from the perspective of the accelerating observer, all parts of the body interact simultaneously with the force carriers of electromagnetic interaction the photons. Of course, the simultaneity of interactions is valid up to a certain approximation precision. The interactions at the leading and trailing ends of an accelerating rod may not be exactly synchronized in the rest frame of the rod and show very small time differences. Therefore, the proper length of the rod may undergo minor oscillations. However, we assume that on average the interactions are simultaneous and the proper length of the rod does not change. (ii) Our second basic assumption is that acceleration is not continuous, but occurs in very small instantaneous velocity boosts. This assumption makes sense when we consider the acceleration of a classical body. According to quantum field theory interactions occur through the exchange of field quanta. Such a field exchange provides a continuous transfer of energy. Therefore, at the quantum level, our second assumption does not seem correct. On the other hand, the acceleration of a classical body requires numerous interactions over a relatively long time interval. Although each interaction provides a continuous transfer of energy, each transferred energy is almost infinitesimal on the classical scale. Therefore, it would be a good approximation to assume that a classical body is

\footnotetext{
${ }^{1}$ This does not mean that the clock ticks backwards in time. However, the trailing clock ticks slower during acceleration than the clock at the front, and at the end of the process it lagged behind.

${ }^{2}$ Some authors argue that clock synchronization is simply a matter of convention [7] and a theory maintaining absolute simultaneity is equivalent to special theory of relativity [8]. But if clock desynchronization is obtained as a result of the acceleration procedure, in the amount predicted by the special theory of relativity, these claims appear to be false. However, the issue is much more complicated than it seems and requires a detailed examination. It goes beyond our purpose in this article and will be explored in our forthcoming paper.
} 
accelerated by an innumerable sequence of instantaneous acceleration steps. ${ }^{3}$

\section{Contraction of rods and desynchronization of clocks}

Consider a rod of proper lenght $\ell$ accelerating according to our basic assumptions (i) and (ii). In Ref.[9] under similar assumptions, it was shown that the length of the rod observed from an inertial frame contracts by an amount exactly equal to the Lorentz contraction factor. Indeed, for simultaneous speed boosts in the accelerating frame of rod, rear boost precedes front boost in the inertial frame $S$ by a time interval $\Delta t=\beta \gamma \ell / c$. Speed of rear end of the rod exceeds speed of front end by $d v$ during $\Delta t$ and the rod is contracted by an amount $d L=d v \Delta t=\beta \gamma \ell d \beta$. Suppose the rod is initially at rest in inertial frame $S$. Then it accelerates to a final velocity $v$. The total amount of contraction is given by the following integral [9]:

$$
\Delta L=\ell \int_{0}^{\beta} \frac{\beta^{\prime}}{\sqrt{1-\beta^{\prime 2}}} d \beta^{\prime}=\ell\left(1-\frac{1}{\gamma}\right)
$$

Hence, after the acceleration procedure is finished, the observer in the inertial frame $S$ observes a moving rod of lenght $L^{\prime}=\ell-\Delta L=\ell / \gamma$. Although the rear and front end points of the rod were considered during the calculation, it can be shown that a similar contraction will occur for any two points on the rod.

For those who follow Einstein's approach, this result is one of many examples demonstrating the consistency of special relativity. According to this approach, Lorentz contraction is imposed as a rule; the laws of interactions and motion must give a result consistent with this rule. On the other hand, according to our approach, result (2.1) is the cause of the Lorentz contraction: The rigid structure of the body is provided by the interactions between the particles that make up the body. If we consider the EM interaction acting on the classical scale, force carriers photons propagate at the speed of light. As a result of the speed of light being constant in different reference frames, the difference of interaction times of photons at two different points shift between these reference frames by the amount of $\Delta t=\beta \gamma \Delta \ell / c .{ }^{4}$ Consequently, the reasoning leading us to eqn.(2.1) is valid and the length of the rod is contracted as given in eqn.(2.1). Admittedly, there are some problems with accepting the process leading to eqn.(2.1) as the cause of Lorentz length contraction. For example, the first question that comes to mind might be the following: Lorentz contraction should also apply to elementary particles such as electrons. Can assumptions (i) and (ii) hold true at the scale of elementary particles? As we discussed earlier, according to quantum field theory, continuous energy transfer can be realized by elementary particle interactions. For this reason, the validity of assumption (ii) at the elementary particle scale requires a new hypothesis. We will briefly address this issue in the next section. Our main purpose in this article is to explain the relativistic desynchronization effect between moving clocks, and we aimed to give the length contraction as an example to make our approach concrete. Einstein's approach states that a moving rod contracts in length compared to its proper length. But it does not say how this happened. As the rod accelerates does the rear end move closer to the front end? Or does the front end approach towards the rear? Or do both ends move towards each other?

\footnotetext{
${ }^{3}$ This is similar to the acceleration of a metal sphere by a high-intensity light beam. When a single photon interacts with a metal surface, the transferred energy is continuous; the velocity of the metal sphere changes continuously from an initial value of $v_{i}$ to a final value of $v_{f}$. But a single photon interacts in a time interval of $\Delta t=\lambda / c$, which is very tiny compared to classical scale. It is therefore a good approximation to assume that the metal sphere is accelerated by a sequence of infinitesimal acceleration steps.

${ }^{4}$ Of course, we do not claim that the two ends of the rod interact. However, we assume that the contraction-causing interactions occur on a very small scale, but still on a scale for which our assumptions (i) and (ii) are valid. Accordingly, the contraction of the rod occurs cumulatively as a sum of contractions on a much smaller scale.
} 
Einstein's approach does not answer these questions. On the other hand, if the length contraction occurs as a result of assumptions (i) and (ii), the rear end of the rod moves towards the forward end during acceleration, causing contraction. ${ }^{5}$

Now, let's come to our main goal in this article: the desynchronization problem of moving clocks. Consider two clocks say, A (rear clock) and B (front clock) separated by a proper length $\ell$. Suppose clocks A and B are rigidly accelerated by small (almost infinitesimal) discrete simultaneous acceleration steps relative to an observer in the frame of the clocks $S^{\prime}$. The clocks are initially at rest and synchronized in an inertial frame $S_{0}$. Then, they accelerate and come to rest in any other inertial frame $S_{1}$ with a velocity $v_{0}$ relative to $S_{0}$. According to an observer in $S_{0}$ frame, for each acceleration step, clock A accelerates $\Delta t=\beta \gamma \ell / c$ before clock B. Speed of clock A exceeds speed of clock B by $d v$ during $\Delta t$ and it undergoes more relativistic time dilation. Consequently, the proper time of clocks shift by

$$
\delta \tau=\tau_{B}-\tau_{A}=(\beta \gamma \ell / c)\left[\sqrt{1-\frac{v^{2}}{c^{2}}}-\sqrt{1-\frac{(v+d v)^{2}}{c^{2}}}\right]
$$

for each acceleration step. If we expand the Taylor series and neglect the terms of order $d \beta^{2}$ we get

$$
\delta \tau=\frac{\ell \beta^{2} \gamma^{2}}{c} d \beta
$$

Integrating eqn.(2.3) from 0 to $\beta_{0}=v_{0} / c$ we get the following time shift:

$$
\Delta \tau=\frac{\ell}{c} \int_{0}^{\beta_{0}} \frac{\beta^{2}}{1-\beta^{2}} d \beta=\frac{\ell}{c}\left[\frac{1}{2} \ln \left(\frac{1+\beta_{0}}{1-\beta_{0}}\right)-\beta_{0}\right] .
$$

Thus, clock A has shifted backwards from clock B by $\Delta \tau$. However, this value differs from special relativistic time shift both in sign and magnitude. Indeed, according to special relativity, clock $\mathrm{A}$ shifts forward from clock B by proper time of $\Delta t_{0} / \gamma_{0}=\beta_{0} \ell / c$.

In fact, the expression (2.4) is compatible with special relativistic time shift. To see this, we need to consider the gravitational redshift observed by the accelerating frame of clocks $S^{\prime}$. Let $g$ be the acceleration observed in the $S^{\prime}$ frame of reference. The coordinate transformation between $S$ and $S^{\prime}$ frames is given by [11]

$$
\begin{aligned}
& t=\left[\frac{c}{g}+\frac{x^{\prime}}{c}\right] \sinh \left(\frac{g t^{\prime}}{c}\right) \\
& x=\left[\frac{c^{2}}{g}+x^{\prime}\right] \cosh \left(\frac{g t^{\prime}}{c}\right)-\frac{c^{2}}{g} \\
& y=y^{\prime} \\
& z=z^{\prime}
\end{aligned}
$$

where we assume that the velocity of $S^{\prime}$ with respect to $S$ is along positive $x$-axis. Therefore, the accelerating observer $S^{\prime}$ uses the following metric:

$$
d s^{2}=-\left(1+\frac{g x^{\prime}}{c^{2}}\right)^{2} c^{2} d t^{\prime 2}+d x^{\prime 2}+d y^{\prime 2}+d z^{\prime 2} .
$$

\footnotetext{
${ }^{5}$ Some authors consider a simultaneous acceleration procedure in the laboratory frame [10]. If, instead of assumption (i), we assume that such an acceleration procedure is valid at the most fundamental level, then the proper length of the rod will extend by a factor of $\gamma$. Such acceleration is not rigid. But, Lorentz transformations and Einstein's approach do not exclude such a possibility; Lorentz transformations impose a relational factor between moving and proper lengths. On the other hand, non-rigid acceleration does not seem correct for various reasons that we cannot address here.
} 
According to Einstein's equivalence principle, the observer $S^{\prime}$ thinks that her frame is not accelerating, but is in a gravitational field described by the metric (2.6). The proper time of $S^{\prime}$ is coordinate dependent: $d \tau=\left(1+\frac{g x^{\prime}}{c^{2}}\right) d t^{\prime}$. Without loss of generality, let's take two points $x_{1}^{\prime}$ and $x_{2}^{\prime}$ on $S^{\prime}$ with $x_{2}^{\prime}>x_{1}^{\prime}$ and $y_{1,2}^{\prime}=z_{1,2}^{\prime}=0$. Then we get the following gravitational time dilation formula:

$$
\frac{\Delta \tau_{1}}{1+\frac{g x_{1}^{\prime}}{c^{2}}}=\frac{\Delta \tau_{2}}{1+\frac{g x_{2}^{\prime}}{c^{2}}} .
$$

Eqn.(2.7) is an exact result for the gravitational redshift in the $S^{\prime}$ frame $[12,13,14]$. Now, let's place the rear clock $\mathrm{A}$ at the origin of $S^{\prime}$, i.e. $x_{A}^{\prime}=0$. Then, the front clock B is at $x_{B}^{\prime}=\ell$. In this case, the following gravitational time dilation relation between clocks $\mathrm{A}$ and $\mathrm{B}$ is obtained:

$$
\Delta \tau_{B}=\left(1+\frac{g \ell}{c^{2}}\right) \Delta \tau_{A}
$$

Suppose that the whole acceleration procedure takes $\tau_{A}$ time relative to an observer at the origin of $S^{\prime}$. Accordingly, at the end of the acceleration, clock B will advance by $\tau_{B}=\left(1+\frac{g \ell}{c^{2}}\right) \tau_{A}$. Therefore, when the acceleration procedure is complete, an observer in the inertial frame $S_{1}$ will observe that clock A lags behind clock B by

$$
\tau_{B}-\tau_{A}=\frac{g \ell}{c^{2}} \tau_{A}
$$

Let us compare the amount of time shifts (2.4) and (2.9) between clocks A and B observed by the inertial frames $S_{0}$ and $S_{1}$. If clocks A and B were synchronized in frame $S_{1}$, the observer in frame $S_{0}$ would find $\Delta \tau=\beta_{0} \ell / c$ instead of (2.4). Thus, to find the desynchronization observed by $S_{0}$ between the clocks, we must subtract (2.9) from (2.4):

$$
\Delta^{\prime} \tau=\frac{\ell}{2 c} \ln \left(\frac{1+\beta_{0}}{1-\beta_{0}}\right)-\frac{\beta_{0} \ell}{c}-\frac{g \ell}{c^{2}} \tau_{A}
$$

Here, $\Delta^{\prime} \tau$ is the proper time shift of clocks observed by $S_{0}$ relative to $S_{1}$. To simplify eqn.(2.10), we make use of transformations (2.5). From eqn.(2.5), we get

$$
t^{\prime}=\frac{c}{g} \tanh ^{-1}(\beta)
$$

where, $t^{\prime}$ is the time it takes for $S^{\prime}$ to reach its velocity $v=\beta c$ (relative to $S_{0}$ ) with respect to a clock ticking at the origin of $S^{\prime}$. Since the whole acceleration procedure takes $\tau_{A}$ time relative to an observer at the origin of $S^{\prime}$ (this is also equal to the total time clock A ticked during acceleration), $t^{\prime}=\tau_{A}$ gives $\beta_{0}=\tanh \left(\frac{g \tau_{A}}{c}\right) \Rightarrow \ln \left(\frac{1+\beta_{0}}{1-\beta_{0}}\right)=\frac{2 g \tau_{A}}{c}$. Hence, the first and third terms of (2.10) cancel each other and we obtain the special relativistic time shift

$$
\Delta^{\prime} \tau=-\frac{\beta_{0} \ell}{c} .
$$

The minus sign in (2.12) indicates that clock A shifts forward from clock B as expected from special relativity.

Now we are in a position to discuss SCH in special relativity. According to SCH the clocks A and B which are initially synchronized in $S_{0}$ maintain their synchronization when they come to rest in $S_{1}$ [3]. If we take the point of view of the observer $S_{0}$, clock A undergoes a special relativistic time shift 
due to acceleration; if the speed increases from $v$ to $v+\Delta v$, clock A will shift forward by $\ell \Delta v / c^{2}$ from clock B. Therefore, clock A advance a number of ticks $\Delta N \simeq \ell \Delta v / T c^{2}$ where $T$ is the period of the clock. Consequently, during acceleration the frequency of the rear clock A increases by an amount $\Delta \nu \simeq \ell a / T c^{2}$ relative to that of the leading clock B as seen by the observer $S_{0}$ [3]. But observer $S_{0}$ also observes a Doppler shift for a light wave sent from B to A. When the light wave reaches A, it is redshifted enough to compensate for the frequency shift $\Delta \nu \simeq \ell a / T c^{2}$ due to special relativistic clock desynchronization. ${ }^{6}$ These two effects cancel each other. Hence, if we assume that clocks A and B are a Mössbauer receiver and source then they will resonantly interact during the acceleration.

The described above is a brief summary of the argument of Ref.[3]. It seems correct when we adopt the Einstein's approach. Indeed, we can interpret the clocks' desynchronization with acceleration as a forward shift in the time of the rear clock. On the other hand, on the basis of assumptions (i) and (ii), we show that the rear clock actually shifted backwards with respect to the front clock. But, due to Einstein's equivalence principle the accelerating observer feels a gravitational field and observes that the rear clock undergoes a gravitational redshift relative to the front clock. This gravitational "backward-time shift" is larger than the time shift for the rigid acceleration and the difference is exactly equal to the special relativistic shift. Therefore, Einstein's equivalence principle and gravitational redshift is necessary to explain special relativistic clock desynchronization.

Of course, the validity of assumptions (i) and (ii) is questionable. In this paper we consider classical clocks. For example, the clocks can be modeled with rotating wheels so that one period of the wheel represents a unit of time. As we have discussed in the introduction, it is a good approximation to assume that a classical body is accelerated by an innumerable sequence of instantaneous acceleration steps. Therefore, assumption (ii) is plausible for classical clocks. Assumption (i) is valid for a rigid acceleration by definition. It is also possible to consider some non-rigid acceleration procedures. One such example is the simultaneous acceleration procedure in the laboratory frame. Such an acceleration procedure can be constructed artificially, for example like the one in the Dewan-Beran-Bell spaceship gedankenexperiment $[1,10]$. However, if we accept that such an acceleration procedure is fundamentally valid in nature, some problems may arise. One problem is to find the preferred inertial frame in which the interactions will occur simultaneously. On the other hand according to assumption (i) there is no particular preferred inertial frame of reference in which the laws of physics are defined. But each body's or particle's rest frame is, in a sense, a preferred frame; accelerating-interactions occur simultaneously in that frame.

\section{Some further restrictions-why should acceleration be discontinu- ous?}

We have a hidden assumption that we use both in the derivation of the length contraction (2.1) and in the derivation of the time shift (2.4). We assume that each small $\Delta v$ acceleration steps occur intermittently from each other, with time intervals of at least $\Delta t=\beta \gamma \ell / c$ relative to the inertial observer $S_{0}$. Otherwise, the clock A will accelerate a second, third, ... times, before it reaches the distance $L^{\prime}=\ell / \gamma$ predicted in the Lorentz contraction formula. If we consider that the acceleration takes place instantaneously with the $\Delta t_{a}$ time intervals in the accelerating frame $S^{\prime}$, then we impose the condition $\Delta t_{a} \geq \beta \ell / c$. For $0 \leq \beta<1$ we get $\Delta t_{a} \geq \ell / c$.

Let's ignore this condition for a moment and assume that acceleration occurs continuously. This is contrary to our assumption (ii). Nevertheless, let's assume that assumption (i) still holds, i.e.

\footnotetext{
${ }^{6}$ This statement is not exact, but it is valid at a certain level of approximation. In Ref.[3] author carry all calculations only to second order in $v / c$
} 
acceleration is rigid. In this case, an observer on one of the clocks, for example on clock A, will observe that clock B remains at rest during acceleration. Therefore, an accelerating frame of reference $S^{\prime}$ can be defined in which both clocks are stationary. On the other hand, according to an observer in the $S_{0}$ frame, the clocks $\mathrm{A}$ and $\mathrm{B}$ are not at rest relative to each other during acceleration. For this reason, the $S^{\prime}$ frame is not well-defined for an observer in the $S_{0}$ frame of reference; is frame $S^{\prime}$ the stationary frame of clock A? Or is it the stationary frame of the clock B? This might be considered as an spurious discussion. But it is not so, because it is an important discussion in determining both length contraction and clock desynchronization. For example, let's say clocks A and B are in an inertial frame $S_{1}$ moving with a velocity $v_{0}$ with respect to frame $S_{0}$ and then they are accelerated for a short time $\Delta t_{a}<\beta_{0} \ell / c$. According to the $S_{0}$ frame, A will start accelerating $\beta_{0} \gamma_{0} \ell / c$ before B. But from the moment A starts to accelerate, the rest frame of the clocks is no longer $S_{1}$. The speed of A varies according to the formula

$$
v(t)=\frac{g\left(t+t_{1}\right)}{\sqrt{1+\frac{g^{2}\left(t+t_{1}\right)^{2}}{c^{2}}}}
$$

where, $t_{1}=\frac{v_{0}}{g \sqrt{1-v_{0}^{2} / c^{2}}}$. Meanwhile $\mathrm{B}$ is moving with a constant velocity $v_{0}$. So what value should we use as the acceleration time difference between $\mathrm{A}$ and $\mathrm{B}$ ? $\beta(t) \gamma(t) \ell / c$ or $\beta_{0} \gamma_{0} \ell / c$ ? This issue remains ambiguous because the $S^{\prime}$ frame is not well-defined relative to $S_{0}$. The above discussion shows that if the length contraction and clock desynchronization are obtained as a result of the acceleration procedure in the amount predicted by the special theory of relativity, then $\Delta t_{a} \geq \ell / c$ condition must be satisfied. Of course, different acceleration procedures are also conceivable. But the problem that the stationary frame of the clocks is not well-defined remains unresolved also in the case of non-rigid acceleration.

Finally, let's discuss the validity of assumption (ii) on the scale of elementary particles. As we have discussed earlier, this requires a new hypothesis. But, is it a reasonable hypothesis? In quantum field theory, although free plane waves are Dirac delta normalized and their momenta can take continuous values, this feature of the theory gives an impression of approximation. For instance, instead of Dirac delta normalization sometimes box normalization is used. In the case of box normalization, the continuous integrals over momentum are replaced by discrete sums [15]. We then take the limit $L \rightarrow \infty$ where $L$ is the size of the box. But this requires the universe to be infinitely large, which is a very accurate approximation that is used a lot in theoretical physics. Consequently, we may expect that the momentum is discrete at a very small scale (probably near the Planck scale) below the elementary particle scale. If so, our assumption (ii) is valid at the elementary particle scale. Our second motivation that the acceleration should be discrete near the Planck scale is based on the algorithmic information theoretical explanation of nature. There are proposals that the universe and everything in it could be encoded as a long (perhaps infinite) string of $0 \mathrm{~s}$ and $1 \mathrm{~s}$, and that the apparent laws of nature could be explained by the evolution of this long string of bits $[16,17]$. If such claims are true, the smallest change in the particle's state must be described with at least 1 bit of information; states evolve discontinuously at the most fundamental level.

\section{References}

[1] J.S. Bell, Speakable and unspeakable in quantum mechanics, Cambridge University Press, Cambridge, 1987 (Section 9: How to teach special relativity).

[2] J. Barbour, The End Of Time, Oxford University Press, Oxford, 1999. 
[3] F. V. Kowalski, Phase-invariant clock hypothesis for accelerating systems, Phys. Rev. A 46, 22612264 (1992).

[4] Meng Guangda et al., Comment on 'Phase-invariant clock hypothesis for accelerating systems', Phys. Rev. A 53, 4605-4606 (1996).

[5] F. V. Kowalski, Reply to 'Comment on 'Phase-invariant clock hypothesis for accelerating systems' , Phys. Rev. A 53, 4607-4608 (1996).

[6] F. V. Kowalski, Accelerating light clocks, Phys. Rev. A 53, 3761-3766 (1996).

[7] H. Reichenbach, The Philosophy of Space and time, Dover Publications, New York 1958.

[8] R. Mansouri and R. U. Sexl, Test Theory of Special Relativity: I. Simultaneity and Clock Synchronization Gen. Rel. Grav. 8, 497-513 (1977).

[9] E. F. Taylor, and A. P. French, Limitation on proper length in special relativity, Am. J. Phys. 51, 889 (1983).

[10] E. Dewan and M. Beran, Note on stress effects due to relativistic contraction, Am. J. Phys. 27, 517 (1959).

[11] Charles W. Misner, Kip S. Thorne, and John Archibald Wheeler, Gravitation, W. H. Freeman and Company, San Francisco, 1973.

[12] W. Moreau, Nonlocality in frequency measurements of uniformly accelerating observers, Am. J. Phys. 60, 561 (1992).

[13] W. Cochran, Some results on the relativistic Doppler effect for accelerated motion, Am. J. Phys. 57, 1039 (1989).

[14] P.T. Landsberg and N.T. Bishop, Gravitational redshift and the equivalence principle, Found. Phys. 6, 727-737 (1976).

[15] W. Greiner and J. Reinhardt, Field Quantization Springer-Verlag, Berlin, Heidelberg 1996.

[16] M. P. Muller Law without law: from observer states to physics via algorithmic information theory, Quantum 4, 301 (2020); arXiv:1712.01826 [quant-ph].

[17] I. Sahin, Comments on indeterminism and undecidability, arXiv: 2106.08132; preprints202106.0056.v1. 